Sławomir Kolasiński, Marta Szumańska

\title{
Minimal Hölder regularity implying finiteness of integral Menger curvature
}

Received: 25 January 2012 / Accepted: 17 April 2012

Published online: 28 June 2012

Abstract. We study two kinds of integral Menger-type curvatures. We find a threshold value of $\alpha_{0}$, a Hölder exponent, such that for all $\alpha>\alpha_{0}$ embedded $C^{1, \alpha}$ manifolds have finite curvature. We also give an example of a $C^{1, \alpha_{0}}$ injective curve and higher dimensional embedded manifolds with unbounded curvature.

\section{Introduction}

Geometric curvature energies are functionals defined on submanifolds of an Euclidean space, whose values can be considered as "total curvature" of the set. We study two families of such functionals. First of them, defined for one dimensional sets, is a generalization of a notion of total Menger curvature introduced by Melnikov in [12] and it is defined as

$$
\mathcal{M}_{p}(\gamma):=\int_{\gamma} \int_{\gamma} \int_{\gamma} R^{-p}(x, y, z) d \mathcal{H}_{x}^{1} d \mathcal{H}_{y}^{1} d \mathcal{H}_{z}^{1}
$$

where $R^{-1}(x, y, z)$ is the Menger curvature of a triple $(x, y, z)$, i.e. the inverse of the radius of the smallest circle passing through $x, y$ and $z$ (cf. Definition 2.1). Throughout this paper we use this notion for a special class of sets i.e. rectifiable curves and for $p \in(2, \infty)$. For $m$-dimensional manifolds $\Sigma \subseteq \mathbb{R}^{n}$ another family of geometric integral curvatures is used, namely

$$
\mathcal{E}_{p}(\Sigma)=\int_{\Sigma^{m+2}} \mathcal{K}\left(\mathbf{x}_{0}, \ldots, \mathbf{x}_{m+1}\right)^{p} d \mathcal{H}_{\mathbf{x}_{0}}^{m} \cdots d \mathcal{H}_{\mathbf{x}_{m+1}^{m}}^{m}, \quad \Sigma^{m+2}=\underbrace{\Sigma \times \cdots \times \Sigma}_{(m+2) \text { times }},
$$

where $\mathcal{K}$ is an analogue of the Menger curvature in higher dimensions (see Definition 2.2).

In this article we give a direct and elementary proof of the following

Theorem. (1) If the arc length parametrization of $\gamma$ is an injective $C^{1, \alpha}$ function for some $\alpha>1-\frac{2}{p}$, then $\mathcal{M}_{p}(\gamma)$ is finite.

S. Kolasiński (凶): M. Szumańska: Institute of Mathematics, University of Warsaw, Banacha 2, Warsaw 02-097, Poland. e-mail: s.kolasinski@mimuw.edu.pl

M. Szumańska: e-mail: m.szumanska@mimuw.edu.pl

Mathematics Subject Classification (1991): Primary: 49Q10; Secondary: 28A75, 49Q20, 49Q15 
(2) If $\alpha>1-\frac{m(m+1)}{p}$ and $\Sigma$ is compact and $C^{1, \alpha}$ regular then $\mathcal{E}_{p}(\Sigma)$ is finite.

Moreover, $\alpha_{0}=1-\frac{m(m+1)}{p}$ is the minimal Hölder exponent above which the energies $\mathcal{M}_{p}$ and $\mathcal{E}_{p}$ become finite.

While working on this article Simon Blatt provided us with his result [2] characterizing $C^{1}$-curves $\gamma$ with $\mathcal{M}_{p}(\gamma)<\infty$ for some $p>3$ exactly as curves having arc-length parametrization in the Sobolev-Slobodeckij space $W^{2-2 / p, p}$. His result implies part (1) of our Theorem due to the embedding $C^{1, \alpha}((0,1)) \subseteq$ $W^{2-2 / p, p}((0,1))$ for $\alpha>\alpha_{0}:=1-2 / p$. Later, as the result of cooperation of the first author with Blatt [3], a second result was achieved - a full characterization of $m$ dimensional, embedded $C^{1}$-manifolds $\Sigma$ with $\mathcal{E}_{p}(\Sigma)<\infty$ for some $p>m(m+1)$ as having local graph representations in $W^{1+s, p}$, where $s=1-m(m+1) / p$. Since $C^{1, \alpha}\left(\mathbb{R}^{m}\right) \subseteq W^{1+s, p}\left(\mathbb{R}^{m}\right)$ for $\alpha>\alpha_{1}:=1-m(m+1) / p$ the result of [3] implies also part (2) of our Theorem. Nevertheless, our work was done independently and we employed completely different techniques. Our proof omits the use of SobolevSlobodeckij and Besov spaces, embedding theorems for these objects and interpolation theory. Instead, we apply elementary methods from calculus and geometry. To prove the second part we construct specific examples of a $C^{1,1-2 / p}$ curve $\gamma$ and a $C^{1,1-m(m+1) / p} m$-manifold $\Sigma$ for which $\mathcal{M}_{p}(\gamma)=\infty$ and $\mathcal{E}_{p}(\Sigma)=\infty$.

The functional $\mathcal{M}_{2}$ proved to be useful in harmonic analysis. Using roughly the same formula, one can define $\mathcal{M}_{p}$ for any Borel set $E$. David and Léger [8] showed that 1-dimensional sets with finite $\mathcal{M}_{2}$ total curvature are 1-rectifiable. This was a crucial step in the proof of Vitushkin's conjecture and allowed to fully characterize removable sets for bounded analytic functions. Surveys of Mattila [11] and Tolsa [22] explain in more detail the connection between these subjects.

The need to study energies like $\mathcal{M}_{p}$ and $\mathcal{E}_{p}$ grew out from natural sciences. Finiteness of $\mathcal{M}_{p}(\gamma)$ or $\mathcal{E}_{p}(\Sigma)$ enforces higher regularity of $\gamma$ or $\Sigma$ and excludes self-intersections (see $[7,19])$. These properties make curvature energies extremely useful in modeling long, entangled objects like DNA molecules, protein structures or polymer chains; see for example the paper by Banavar et al. [1] or the book by Sutton and Balluffi [21] and the references therein. In this context, functionals similar to $\mathcal{M}_{p}$ were studied earlier by Gonzalez and Maddocks [5]. The authors suggest also the use of $\mathcal{M}_{p}$ as so called knot energy, i.e. an energy which separates knot types by infinite energy barriers. In [5] the authors introduce the notions of the global radius of curvature and the thickness of a curve $\gamma$, by taking the supremum of $R^{-1}(x, y, z)$ over all $x, y, z \in \gamma$. Gonzalez et al. [6] proved that rectifiable curves with finite thickness are $C^{1,1}$ regular.

Functionals similar to $\mathcal{M}_{p}$, where one or two integrations were replaced by taking the supremum, were examined in $[18,14]$. A 2-dimensional close analogue of $\mathcal{E}_{p}$ was also studied by Strzelecki and von der Mosel [15]. Yet another example is the tangent-point energy defined by integrating the radius of a circle passing through one point of a curve and tangent to the curve at some other point. Properties of curves with finite tangent-point energy were investigated by Sullivan [20] (in $C^{2}$ case) and by Strzelecki and von der Mosel [17] (in continuous case). A similar tangent-point energy in higher dimensions was also studied in [16]. In all cases, finiteness of these functionals (for sufficiently large $p$ ) implies self-avoidance and 
smoothing effects. Moreover one can use some of these energies to apply topological constraints in variational problems. In [15, Section 7] Strzelecki and von der Mosel proved existence of area minimizing surfaces in a given isotopy class under the constraint of bounded curvature.

Lerman and Whitehouse $[9,10]$ suggested a whole class of curvature energies for higher dimensional objects. However, the integrands in their definitions scale differently and it seems that these energies can not serve our needs. Nevertheless, the authors proved [9, Theorems 1.2 and 1.3] that their integral curvatures can be used to characterize $d$-dimensional rectifiable measures in the sense of David and Semmes [4].

Remark 1.1. We shall use a letter $C$ to denote a general constant, whose value may change from line to line even in one series of transformations.

\section{Preliminaries}

In this section we introduce the notation that will be used throughout the paper. We also explain the relations between two types of energies we consider.

We use the symbol $\mathbb{B}(x, r)$ for the ball with radius $r$ centered at $x$, while we write $\mathbb{B}_{r}$ if the ball is centered at the origin and $\mathbb{B}_{r}^{m}$, when we additionally want to emphasize the dimension of the ball. We denote the m-dimensional Hausdorff measure by $\mathcal{H}^{m}$ and $T_{a} \Sigma$ denotes the vector space tangent to $\Sigma$ at the point $a$. The symbol $S_{L}$ stands for the circle of length $L$, i.e. $S_{L}=\mathbb{R} / L \mathbb{Z}$. For $U \subseteq \mathbb{R}^{n}$, a vector space, and $f: U \rightarrow U^{\perp}$ a function, we set

$$
\operatorname{Graph}(f)=\left\{(x, f(x)) \in \mathbb{R}^{n}: x \in U\right\} .
$$

For a tuple $T=\left(\mathbf{x}_{0}, \ldots, \mathbf{x}_{k}\right)$ of $(k+1)$ points in $\mathbb{R}^{n}$, we write $\triangle T$ to denote the convex hull of the set $\left\{\mathbf{x}_{0}, \mathbf{x}_{1}, \ldots, \mathbf{x}_{k}\right\}$, i.e. the smallest convex subset of $\mathbb{R}^{n}$ which contains all the points $\mathbf{x}_{0}, \ldots, \mathbf{x}_{k}$. Typically $\triangle T$ will just be a $k$-simplex (a triangle for $k=2$ and a tetrahedron for $k=3$ ).

As we mentioned in the introduction, Menger curvature of three points is a reciprocal of the radius of the smallest circle passing through these points. Using the sine theorem, we can define it as follows

Definition 2.1. Let $x_{0}, x_{1}, x_{2}$ be three points in $\mathbb{R}^{n}$. The radius of Menger curvature of $x_{0}, x_{1}$ and $x_{2}$ is given by the formula

$$
R\left(x_{0}, x_{1}, x_{2}\right)=\frac{\left|x_{1}-x_{0}\right|\left|x_{2}-x_{0}\right|\left|x_{2}-x_{1}\right|}{4 \mathcal{H}^{2}\left(\triangle\left(x_{0}, x_{1}, x_{2}\right)\right)} .
$$

We can use the above definition to compare Menger curvature and its higher dimensional generalizations. ${ }^{1}$ In this paper we use integral curvature functional $\mathcal{E}_{p}$ defined in [7] whose integrand is the $p$ th power of the discrete curvature $\mathcal{K}$ given by

\footnotetext{
${ }^{1}$ Natural generalization of Menger curvature of three points would be the inverse of the radius of the sphere passing through four points. However this definition is not rewarding for integral curvature energies-see [15, Appendix B].
} 
Definition 2.2. Let $T=\left(\mathbf{x}_{0}, \ldots, \mathbf{x}_{m+1}\right)$ be an $(m+2)$-tuple of points in $\mathbb{R}^{n}$. The discrete curvature of $T$ is given by the formula

$$
\mathcal{K}(T)=\frac{\mathcal{H}^{m+1}(\triangle T)}{\operatorname{diam}(T)^{m+2}} .
$$

Now we introduce the definition of our functional.

Definition 2.3. Let $\Sigma \subseteq \mathbb{R}^{n}$ be some $m$-dimensional subset of $\mathbb{R}^{n}$. We define the p-energy of $\Sigma$ by the formula

$$
\mathcal{E}_{p}(\Sigma)=\int_{\Sigma^{m+2}} \mathcal{K}\left(\mathbf{x}_{0}, \ldots, \mathbf{x}_{m+1}\right)^{p} d \mathcal{H}_{\mathbf{x}_{0}}^{m} \cdots d \mathcal{H}_{\mathbf{x}_{m+1}^{m}}^{m} .
$$

The quantity $\mathcal{K}(T)$ should be seen as a generalization of the Menger curvature to higher dimensions. It behaves in the same way as $R^{-1}$ under scaling, i.e. $\mathcal{K}(\lambda T)=$ $\lambda^{-1} \mathcal{K}(T)$. Notice that we always have $R^{-1}(x, y, z)>\mathcal{K}(x, y, z)$. Furthermore, for a class of roughly regular triangles $T=(x, y, z)$ (i.e. satisfying $\mathfrak{h}_{\min }(T) \geq$ $\eta \operatorname{diam}(T)$, where $\mathfrak{h}_{\min }(T)$ is the minimal height of $T$ and $\eta \in(0,1]$ is some fixed number) the two quantities $\mathcal{K}(T)$ and $R^{-1}(T)$ are comparable up to a constant depending only on $\eta$. However they are not comparable, when considered on the family of all triangles so we cannot infer finiteness of $\mathcal{M}_{p}(\gamma)$ from finiteness of $\mathcal{E}_{p}(\gamma)$.

The definition of $\mathcal{K}$ is based on another notion of discrete curvature $\mathcal{K}_{\mathrm{SvdM}}$ introduced by Strzelecki and von der Mosel in [15] for 4-tuples of points (tetrahedrons). Yet again, the quantities $\mathcal{K}(T)$ and $\mathcal{K}_{\mathrm{SvdM}}(T)$ are comparable for the class of roughly regular tetrahedrons, i.e. such that $\mathfrak{h}_{\min }(T) \geq \eta \operatorname{diam}(T)$ for some fixed $\eta \in(0,1]$.

In the proofs we will use the Jones' $\beta$-numbers. For a set $E \subset \mathbb{R}^{n}$, for any $x \in \mathbb{R}^{n}$ and $r \in \mathbb{R}$ we define

$$
\beta_{E}^{m}(x, r):=\inf _{H} \sup _{y \in E \cap \mathbb{B}(x, r)} \frac{\operatorname{dist}(y, x+H)}{r},
$$

where the infimum is taken over all $m$-hyperplanes $H$ in the Grassmannian $G(m, n)$. The quantity $\beta_{E}^{m}(x, t)$ measures, in a scale invariant way, how well the set is approximated by hyperplanes in the ball $\mathbb{B}(x, t)$. We omit the indices $E$ and $m$ if the choice of the set and its dimension is clear from the context. The relations between total Menger curvature $\left(\mathcal{M}_{2}\right)$ and double integral of $\beta$-numbers for rectifiable curves and Ahlfors regular sets were investigated by Peter Jones, who never published those results, however they are presented in Herve Pajot's book [13, chapter 3].

\section{Injective $C^{1, \alpha}$ curves have finite $\mathcal{M}_{p}$ curvature}

The main step of the proof of the finiteness of $\mathcal{M}_{p}(\gamma)$ for $C^{1, \alpha}$ curves with $\alpha>$ $1-2 / p$, is to show that one can control the angle between secants on small arcs on the curves. This leads to the estimation of Menger curvature. 
Theorem 3.1. If $\Gamma: S_{L} \rightarrow \mathbb{R}^{3}$ is an injective arc-length parametrization of $\gamma$ and $\Gamma \in C^{1, \alpha}\left(S_{L}\right)$, where $\alpha>1-\frac{2}{p}$ for some $p>2$, then $\mathcal{M}_{p}(\gamma)$ is finite.

To formulate the next lemma we need to introduce geometric objects we will use. Let $C^{+}(P, \vec{v}, \alpha)$ be a "half cone" with a vertex $\mathrm{P}$, an axis parallel to the given vector $\vec{v}$ and an opening angle $\alpha$

$$
C^{+}(P, \vec{v}, \alpha):=\left\{P+x:|\Varangle(\vec{v}, x)|<\frac{\alpha}{2} \text { and } x \neq 0\right\} .
$$

Intersection of two half-cones with vertices $P$ and $Q$, common axis $P Q$ and opening angle $\alpha$ will be denoted as follows

$$
D(P, Q, \alpha):=C^{+}(P, \overrightarrow{P Q}, \alpha) \cap C^{+}(Q, \overrightarrow{Q P}, \alpha)
$$

Now we are ready to formulate and prove the lemma.

Lemma 3.2. Let $\Gamma: S_{L} \rightarrow \mathbb{R}^{3}$ be injective, and

$$
\left|\Gamma^{\prime}(x)-\Gamma^{\prime}(y)\right| \leq \lambda|x-y|^{\alpha} \text { for all } x, y \in S_{L}
$$

Then for any $x, y \in S_{L}$ satisfying $\frac{5}{2} \lambda|x-y|^{\alpha}<1$ the following inclusion holds

$$
\Gamma((x, y)) \subset D\left(\Gamma(x), \Gamma(y), \eta|x-y|^{\alpha}\right),
$$

where $\eta$ is a constant which depends only on $\lambda$.

Proof of Lemma 3.2. Let $x, y, z \in S_{L}$ be such that $x<z<y$. We are going to estimate the angle between two secant lines: one passing through $\Gamma(x)$ and $\Gamma(y)$ and the other one passing through $\Gamma(x)$ and $\Gamma(z)$. First, note that for any two unit vectors $u, v \in S^{n-1}$ forming a small angle $\Varangle(u, v)=\theta$ we have $\theta \simeq|u-v|$. Hence, it suffices to estimate the difference $\frac{\Gamma(x)-\Gamma(y)}{|\Gamma(x)-\Gamma(y)|}$ and $\frac{\Gamma(x)-\Gamma(z)}{|\Gamma(x)-\Gamma(z)|}$. Let us calculate

$$
\begin{aligned}
\left|\frac{\Gamma(x)-\Gamma(y)}{|\Gamma(x)-\Gamma(y)|}-\frac{\Gamma(x)-\Gamma(z)}{|\Gamma(x)-\Gamma(z)|} \leq\right| \frac{\Gamma(x)-\Gamma(y)}{|\Gamma(x)-\Gamma(y)|}-\Gamma^{\prime}(x) \mid \\
+\left|\frac{\Gamma(x)-\Gamma(z)}{|\Gamma(x)-\Gamma(z)|}-\Gamma^{\prime}(z)\right|+\left|\Gamma^{\prime}(x)-\Gamma^{\prime}(z)\right| .
\end{aligned}
$$

We have

$$
\begin{aligned}
\left|\Gamma^{\prime}(x)-\frac{\Gamma(x)-\Gamma(y)}{|\Gamma(x)-\Gamma(y)|}\right| \leq & \left|\Gamma^{\prime}(x)-\frac{\Gamma(x)-\Gamma(y)}{|x-y|}\right| \\
& +\left|\frac{\Gamma(x)-\Gamma(y)}{|x-y|}-\frac{\Gamma(x)-\Gamma(y)}{|\Gamma(x)-\Gamma(y)|}\right| \\
\leq & 2\left|\Gamma^{\prime}(x)-\frac{\Gamma(x)-\Gamma(y)}{|x-y|}\right|,
\end{aligned}
$$

where the last inequality holds, because the distance between vector $v=\frac{\Gamma(x)-\Gamma(y)}{|x-y|}$ and its projection onto the unit sphere $\frac{\Gamma(x)-\Gamma(y)}{|\Gamma(x)-\Gamma(y)|}$ is not greater than the distance 
between $v$ and any arbitrarily chosen unit vector (recall that $\Gamma$ is an arc-length parametrization, so $\left.\left|\Gamma^{\prime}\right| \equiv 1\right)$.

$$
\begin{aligned}
\left|\Gamma^{\prime}(x)-\frac{\Gamma(x)-\Gamma(y)}{|x-y|}\right| & =\left|\Gamma^{\prime}(x)-\frac{1}{y-x} \int_{[x, y]} \Gamma^{\prime}(s) d s\right| \\
& \leq \frac{1}{y-x} \int_{[x, y]}\left|\Gamma^{\prime}(x)-\Gamma^{\prime}(s)\right| d s \leq \frac{1}{y-x} \int_{[x, y]} \lambda(s-x)^{\alpha} d s \\
& \leq \lambda(y-x)^{\alpha} .
\end{aligned}
$$

Combining the above inequalities we obtain

$$
\begin{aligned}
\left|\frac{\Gamma(x)-\Gamma(y)}{|\Gamma(x)-\Gamma(y)|}-\frac{\Gamma(x)-\Gamma(z)}{|\Gamma(x)-\Gamma(z)|}\right| & \leq 2 \lambda|x-y|^{\alpha}+2 \lambda|x-z|^{\alpha}+\lambda|x-z|^{\alpha} \\
& \leq 5 \lambda|x-y|^{\alpha} .
\end{aligned}
$$

This implies that $\Gamma((x, y))$ is included in the cone

$$
C\left(\Gamma(x), \Gamma(y)-\Gamma(x), 2 \arcsin \left(\frac{5}{2} \lambda|x-y|^{\alpha}\right)\right) .
$$

Analogously

$$
\Gamma((x, y)) \in C\left(\Gamma(y), \Gamma(x)-\Gamma(y), 2 \arcsin \left(\frac{5}{2} \lambda|x-y|^{\alpha}\right)\right) .
$$

Thus

$$
\Gamma((x, y)) \in D\left(\Gamma(x), \Gamma(y), \eta|x-y|^{\alpha}\right) .
$$

The lemma proven above gives an estimation for Jones $\beta$-numbers; it is easy to notice that there exists $R_{0}$, such that for $r<R_{0}$ we have

$$
\beta(x, r)<r^{\alpha} .
$$

In case of plane curves it is possible to use the estimation to prove this finiteness of $\mathcal{M}_{p}$, (one can modify the reasoning from [13]), but it is not clear if it is possible to adapt it to curves in $\mathbb{R}^{3}$ and even in 2-space the arguing is long and complicated. Here, Lemma 3.2 gives us additional information. We not only know that the curve is close to a line in a small ball, but we can also point out the line. This information makes the proof of the finiteness of $\mathcal{M}_{p}$ much easier, as the following lemma holds.

Lemma 3.3. Let $\Gamma: S_{L} \rightarrow \mathbb{R}^{3}$ be an injective, arc-length parametrization of a curve $\gamma$ and let $p>2$ and $\alpha>1-\frac{2}{p}$. If there exist constants $\eta$ and $\varepsilon>0$ such that $\eta \varepsilon^{\alpha}<\frac{\pi}{2}$ and for each $|x-y|<\varepsilon$ we have

$$
\Gamma((x, y)) \subset D\left(\Gamma(x), \Gamma(y), \eta|x-y|^{\alpha}\right),
$$

then $\mathcal{M}_{p}(\gamma)<\infty$. 
Proof of Lemma 3.3. We start with a simple geometric observation. If $S \in D(P, Q, \beta)$ and $0<\beta<\frac{\pi}{2}$ then

$$
c(P, Q, S)=\frac{1}{R(P, Q, S)} \leq \frac{2 \sin \beta}{|P Q|} .
$$

Thus, from (1), for $|x-y|<\varepsilon$ and $z \in[x, y]$ we have

$$
c(\Gamma(x), \Gamma(y), \Gamma(z)) \leq \frac{2 \sin \left(\eta|x-y|^{\alpha}\right)}{|\Gamma(x)-\Gamma(y)|} \leq \frac{2 \eta|x-y|^{\alpha}}{|\Gamma(x)-\Gamma(y)|}
$$

As $\Gamma^{\prime}$ is Hölder continuous, $\Gamma$ is locally bi-Lipschitz (see e.g. [18, Lemma 3.2]); using a compactness argument one can show that $\Gamma$ is globally bi-Lipschitz, thus there exists a constant $d>0$ such that

$$
c(\Gamma(x), \Gamma(y), \Gamma(z)) \leq d|x-y|^{\alpha-1} .
$$

Now we are ready to estimate the triple integral

$$
\begin{aligned}
\mathcal{M}_{p}(\gamma)= & \int_{S_{L}} \int_{S_{L}} \int_{S_{L}} c^{p}(\Gamma(x), \Gamma(y), \Gamma(z)) d x d y d z \\
\leq & C \int_{S_{L}} \int_{\left\{y \in S_{L}\right.} \int_{|x-y|<\varepsilon\}} c^{p}(\Gamma(x), \Gamma(y), \Gamma(z)) d z d y d x \\
& +\int_{S_{L}} \int_{\left\{y \in S_{L}\right.} \int_{|| x-y \mid>\varepsilon\}} c^{p}(\Gamma(z), \Gamma(y), \Gamma(x)) d z d y d x \\
\leq & \left.\int_{S_{L}} \int_{\left\{y \in S_{L} \mid\right.}|x-y|^{p(\alpha-1)} \cdot|x-y|<\varepsilon\right\} \\
& +C \int_{S_{L}\left\{y \in S_{L} \mid\right.} \int_{|x-y|>\varepsilon\}} \int_{S_{L}} \varepsilon^{p} d z d y d x \\
\leq & C \int_{S_{L}} \int_{\left\{y \in S_{L}|x-y|<\varepsilon\right\}} \mid x-p+1 d y d x+\text { const }<\infty,
\end{aligned}
$$

as $\alpha>1-\frac{2}{p}$.

The proof of Theorem 3.1 follows immediately from the Lemmas 3.2 and 3.3.

Proof of Lemma 3.1. As $\Gamma$ satisfies assumption of Lemma 3.2, we know that there exists $\varepsilon>0$ such that if $|x-y| \leq \varepsilon$ then

$$
\Gamma((x, y)) \subset D\left(\Gamma(x), \Gamma(y), \mathrm{d}|x-y|^{\alpha}\right),
$$

where $d$ is a constant which depends only on Hölder constant $C$. Thus using Lemma 3.3 we obtain the thesis. 


\section{Manifolds of class $C^{1, \alpha}$ have finite integral curvature}

In this section we prove a counterpart of Theorem 3.1 for $m$-dimensional submanifolds of $\mathbb{R}^{n}$.

Theorem 4.1. Let $p>m(m+1)$ be some number and let $\Sigma \subseteq \mathbb{R}^{n}$ be a compact manifold of class $C^{1, \alpha}$. If $\alpha>1-\frac{m(m+1)}{p}$ then $\mathcal{E}_{p}(\Sigma)$ is finite.

Lemma 4.2. Let $\Sigma \subseteq \mathbb{R}^{n}$ be a compact manifold of class $C^{1, \alpha}$ for some $\alpha \in(0,1)$. Then there exist constants $R=R(\Sigma)>0$ and $C=C(\Sigma)>0$ such that for each $a \in \Sigma$ and each $r \leq R$

$$
\beta(a, r) \leq C r^{\alpha}
$$

Proof. Since $\Sigma$ is compact, we can find a radius $R>0$ and a constant $C>0$ such that for each $a \in \Sigma$ there exists a function $f_{a} \in C^{1, \alpha}\left(T_{a} \Sigma, T_{a} \Sigma^{\perp}\right)$ such that

$$
\Sigma \cap \mathbb{B}(a, R)=\left(a+\operatorname{Graph}\left(f_{a}\right)\right) \cap \mathbb{B}(a, R),
$$

$f_{a}(0)=0, \quad D f_{a}(0)=0 \quad$ and $\forall x, y \in T_{a} \Sigma \quad\left|D f_{a}(x)-D f_{a}(y)\right| \leq C|x-y|^{\alpha}$.

Fix some $a \in \Sigma$ and a radius $r \leq R$. Let $b \in \Sigma \cap \mathbb{B}(a, r)$. Since $\Sigma \cap \mathbb{B}(a, R)$ is the graph of $f_{a}$, there exists a point $x \in T_{a} \Sigma$ such that $b=a+x+f_{a}(x)$. By the fundamental theorem of calculus we have

$$
\begin{aligned}
\left|f_{a}(x)\right| & =\left|\int_{0}^{1} \frac{d}{d t} f_{a}(t x) d t\right| \leq|x| \sup _{y \in T_{a} \Sigma \cap \mathbb{B}_{|x|}}\left|D f_{a}(y)-D f_{a}(0)\right| \\
& \leq C|x|^{1+\alpha} \leq C|b-a|^{1+\alpha} .
\end{aligned}
$$

Note that $\left|f_{a}(x)\right|$ is just the distance of $b$ from the affine plane $a+T_{a} \Sigma$. Hence

$$
\sup _{b \in \Sigma \cap \mathbb{B}(a, r)} \operatorname{dist}\left(b, a+T_{a} \Sigma\right) \leq C r^{1+\alpha}
$$

and we obtain

$$
\begin{aligned}
\beta(a, r) & =\frac{1}{r} \inf _{H \in G(n, m)}\left(\sup _{b \in \Sigma \cap \mathbb{B}(a, r)} \operatorname{dist}(b, a+H)\right) \\
& \leq \frac{1}{r} \sup _{b \in \Sigma \cap \mathbb{B}(a, r)} \operatorname{dist}\left(b, a+T_{a} \Sigma\right) \leq C r^{\alpha} .
\end{aligned}
$$

Lemma 4.3. ([3, Lemma 3.4]) Let $\Sigma \subseteq \mathbb{R}^{n}$ be an m-dimensional manifold. Choose $m+2$ points $\mathbf{x}_{0}, \ldots, \mathbf{x}_{m+1}$ of $\Sigma$ and set $T=\triangle\left(\mathbf{x}_{0}, \ldots, \mathbf{x}_{m+1}\right)$ and $d=\operatorname{diam}(T)$. There exists a constant $C=C(m, n)$ such that

$$
\mathcal{H}^{m+1}(T) \leq C \beta\left(\mathbf{x}_{0}, d\right) d^{m+1}, \text { hence } \mathcal{K}\left(\mathbf{x}_{0}, \ldots, \mathbf{x}_{m+1}\right) \leq C \frac{\beta\left(\mathbf{x}_{0}, d\right)}{d} .
$$


A similar result is proven in [7, Proposition 1.51 and Corollary 1.52]. The proof presented here follows the lines of the proofs presented there. Note that we assumed $\Sigma$ to be a manifold but the proof works also for an arbitrary set $\Sigma$ of Hausdorff dimension $m$ or even for any set.

Proof. If the affine space $\left\{\mathbf{x}_{0}, \ldots, \mathbf{x}_{m+1}\right\}$ is not $(m+1)$-dimensional then $\mathcal{H}^{m+1}(T)=0$ and there is nothing to prove. Hence, we can assume that $T$ is an $(m+1)$-dimensional simplex. The measure $\mathcal{H}^{m+1}(T)$ can be expressed by the formula

$$
\mathcal{H}^{m+1}(T)=\frac{1}{m+1} \operatorname{dist}\left(\mathbf{x}_{m+1},\left\{\mathbf{x}_{0}, \ldots, \mathbf{x}_{m}\right\}\right) \mathcal{H}^{m}\left(\triangle\left(\mathbf{x}_{0}, \ldots, \mathbf{x}_{m}\right)\right) .
$$

In the same way, one can express the measure $\mathcal{H}^{m}\left(\triangle\left(\mathbf{x}_{0}, \ldots, \mathbf{x}_{m}\right)\right)$, so certainly

$$
\mathcal{H}^{m+1}(T) \leq \frac{1}{(m+1) !} d^{m+1}
$$

Hence, if $\beta\left(\mathbf{x}_{0}, d\right)=1$, then there is nothing to prove, so we can assume that $\beta\left(\mathbf{x}_{0}, d\right)<1$.

Due to compactness of the Grassmannian $G(n, m)$ we can find an $m$-plane $H \in G(n, m)$ such that

$$
\sup _{y \in \Sigma \cap \mathbb{B}\left(\mathbf{x}_{0}, d\right)} \operatorname{dist}\left(y, \mathbf{x}_{0}+H\right)=d \beta\left(\mathbf{x}_{0}, d\right) .
$$

Set $h=d \beta\left(\mathbf{x}_{0}, d\right)<d$. Without loss of generality we can assume that $\mathbf{x}_{0}$ lies at the origin. Let us choose an orthonormal coordinate system $v_{1}, \ldots, v_{n}$ such that $H=\operatorname{span}\left\{v_{1}, \ldots, v_{m}\right\}$. Because of (3) in our coordinate system we have

$$
T \subseteq[-d, d]^{m} \times[-h, h]^{n-m} .
$$

Of course $T$ lies in some $(m+1)$-dimensional section of the above product. Let

$$
\begin{aligned}
V & :=\left\{\mathbf{x}_{0}, \ldots, \mathbf{x}_{m+1}\right\}=\operatorname{span}\left\{\mathbf{x}_{1}, \ldots, \mathbf{x}_{m+1}\right\}, \\
Q(a, b) & :=[-a, a]^{m} \times[-b, b]^{n-m}, \\
Q & :=Q(d, h) \\
\text { and } P & :=V \cap Q .
\end{aligned}
$$

Note that all of the sets $V, Q$ and $P$ contain $T$. Choose another orthonormal basis $w_{1}, \ldots, w_{n}$ of $\mathbb{R}^{n}$, such that $V=\operatorname{span}\left\{w_{1}, \ldots, w_{m+1}\right\}$. Set

$$
S:=\left\{x \in V^{\perp}:\left|\left\langle x, w_{i}\right\rangle\right| \leq h \text { for } i=m+2, \ldots, n\right\} .
$$

Observe that $S$ is isometric with the cube $[-h, h]^{n-m-1}$, so diam $S=2 h \sqrt{n-m-1}$ $=: 2 A h$, where $A=A(n, m)=\operatorname{diam}\left([0,1]^{n-m-1}\right)$. In this setting (in the basis $\left.v_{1}, \ldots, v_{n}\right)$ we have

$$
P \times S=P+S \subseteq Q(d+2 h A, h+2 h A) .
$$


Recall that $h=d \beta\left(\mathbf{x}_{0}, d\right)<d$. We obtain the following estimate

$$
\begin{aligned}
\mathcal{H}^{n}(T \times S) & \leq \mathcal{H}^{n}(P \times S) \leq \mathcal{H}^{n}(Q(d+2 h A, h+2 h A)) \\
& \leq(2 d+4 h A)^{m}(2 h+4 h A)^{n-m} \\
& \leq\left(2 d+4 d \beta\left(\mathbf{x}_{0}, d\right) A\right)^{m}\left(2 d \beta\left(\mathbf{x}_{0}, d\right)+4 d \beta\left(\mathbf{x}_{0}, d\right) A\right)^{n-m} \\
& \leq(2+4 A)^{n} d^{n} \beta\left(\mathbf{x}_{0}, d\right)^{n-m} .
\end{aligned}
$$

On the other hand we have

$$
\begin{aligned}
\mathcal{H}^{n}(T \times S) & =\mathcal{H}^{m+1}(T) \mathcal{H}^{n-m-1}(S)=\mathcal{H}^{m+1}(T) 2^{n-m-1} h^{n-m-1} \\
& =2^{n-m-1} \mathcal{H}^{m+1}(T) d^{n-m-1} \beta\left(\mathbf{x}_{0}, d\right)^{n-m-1} .
\end{aligned}
$$

Hence

$$
\begin{aligned}
2^{n-m-1} \mathcal{H}^{m+1} & (T) d^{n-m-1} \beta\left(\mathbf{x}_{0}, d\right)^{n-m-1} \leq(2+4 A)^{n} d^{n} \beta\left(\mathbf{x}_{0}, d\right)^{n-m} \\
& \Longleftrightarrow \mathcal{H}^{m+1}(T) \leq(2+4 A)^{n} 2^{-(n-m-1)} d^{m+1} \beta\left(\mathbf{x}_{0}, d\right) .
\end{aligned}
$$

We may set $C=C(n, m)=(2+4 A)^{n} 2^{-(n-m-1)}$.

Now we can prove the main result of this section.

Proof of Theorem. 4.1. Let

$$
\mu=\underbrace{\mathcal{H}^{m} \otimes \cdots \otimes \mathcal{H}^{m}}_{m+1} .
$$

If $T=\left(x_{0}, x_{1}, \ldots, x_{m+1}\right) \in \Sigma^{m+2}$, we shall write $T=\left(x_{0}, \bar{x}\right)$. Using Lemma 4.3 we obtain

$$
\begin{aligned}
\mathcal{E}_{p}(\Sigma) & =\int_{\Sigma^{m+2}} \mathcal{K}\left(x_{0}, \bar{x}\right)^{p} d \mu(\bar{x}) d \mathcal{H}^{m}\left(x_{0}\right) \\
& \leq \int_{\Sigma} \int_{\Sigma^{m+1}}\left(\frac{\beta\left(x_{0}, \operatorname{diam}\left(x_{0}, \ldots, x_{m+1}\right)\right)}{\operatorname{diam}\left(x_{0}, \ldots, x_{m+1}\right)}\right)^{p} d \mu(\bar{x}) d \mathcal{H}^{m}\left(x_{0}\right) .
\end{aligned}
$$

For $x_{0} \in \Sigma$ and $k \in \mathbb{Z}$ we define the sets

$$
\Sigma_{k}\left(x_{0}\right):=\left\{\left(x_{1}, \ldots, x_{m+1}\right) \in \Sigma^{m+1}: \operatorname{diam}\left(x_{0}, \ldots, x_{m+1}\right) \in\left(2^{-k-1}, 2^{-k}\right]\right\} .
$$

Choose $K_{0} \in \mathbb{Z}$ such that $2^{-K_{0}} \geq 2 \operatorname{diam}(\Sigma)$. Now we can write

$$
\mathcal{E}_{p}(\Sigma) \leq \int_{\Sigma} \sum_{k=K_{0}}^{\infty} \int_{\Sigma_{k}\left(x_{0}\right)}\left(\frac{\beta\left(x_{0}, \operatorname{diam}\left(x_{0}, \bar{x}\right)\right)}{\operatorname{diam}\left(x_{0}, \bar{x}\right)}\right)^{p} d \mu(\bar{x}) d \mathcal{H}^{m}\left(x_{0}\right) .
$$

Fix some small number $\varepsilon>0$. Since $\Sigma$ is compact, we can find a radius $R>0$ such that for each $a \in \Sigma$ there exists a function $f_{a} \in C^{1, \alpha}\left(T_{a} \Sigma, T_{a} \Sigma^{\perp}\right)$ such that

$$
\Sigma \cap \mathbb{B}(a, R)=\left(a+\operatorname{Graph}\left(f_{a}\right)\right) \cap \mathbb{B}(a, R),
$$




$$
f_{a}(0)=0, \quad D f_{a}(0)=0 \quad \text { and } \forall x, y \in \mathbb{B}_{2 R}^{m} \quad\left|f_{a}(x)-f_{a}(y)\right| \leq \varepsilon|x-y| .
$$

For $r<R$ and $x_{0} \in \Sigma$ we have the following estimate

$$
\mathcal{H}^{m}\left(\Sigma \cap \mathbb{B}\left(x_{0}, r\right)\right) \leq(1+\varepsilon)^{m} \mathcal{H}^{m}\left(\left(x_{0}+T_{x_{0}} \Sigma\right) \cap \mathbb{B}\left(x_{0}, r\right)\right)=(1+\varepsilon)^{m} \omega_{m} r^{m} .
$$

Choose $k_{0} \in \mathbb{Z}$ such that $2^{-k_{0}} \leq R$. Then for each $k \geq k_{0}$ we have

$$
\forall x \in \Sigma \quad \mathcal{H}^{m(m+1)}\left(\Sigma_{k}(x)\right) \leq\left(\omega_{m}(1+\varepsilon)^{m} 2^{-k m}\right)^{m+1}=\frac{C(m, \varepsilon)}{2^{k m(m+1)}} .
$$

Of course we have

$$
\begin{aligned}
& \int_{\Sigma} \sum_{k=K_{0}}^{k_{0}-1} \int_{\Sigma_{k}\left(x_{0}\right)}\left(\frac{\beta\left(x_{0}, \operatorname{diam}\left(x_{0}, \bar{x}\right)\right)}{\operatorname{diam}\left(x_{0}, \bar{x}\right)}\right)^{p} d \mu(\bar{x}) d \mathcal{H}^{m}\left(x_{0}\right) \\
& \quad \leq\left(\mathcal{H}^{m}(\Sigma)\right)^{m+2}\left(k_{0}-K_{0}\right) 2^{p k_{0}}<\infty,
\end{aligned}
$$

so to show that $\mathcal{E}_{p}(\Sigma)$ is finite it suffices to estimate the sum from $k_{0}$ to $\infty$. Using Lemma 4.2 and (5) we can write

$$
\begin{aligned}
& \int_{\Sigma} \sum_{k=k_{0}}^{\infty} \int_{\Sigma_{k}\left(x_{0}\right)}\left(\frac{\beta\left(x_{0}, \operatorname{diam}\left(x_{0}, \bar{x}\right)\right)}{\operatorname{diam}\left(x_{0}, \bar{x}\right)}\right)^{p} d \mu(\bar{x}) d \mathcal{H}^{m}\left(x_{0}\right) \\
& \leq C(m, p, \Sigma, \varepsilon) \mathcal{H}^{m}(\Sigma) \sum_{k=k_{0}}^{\infty} \frac{1}{2^{k m(m+1)}}\left(\frac{2^{-k \alpha}}{2^{-k-1}}\right)^{p} \\
& =C^{\prime}(m, p, \Sigma, \varepsilon) \sum_{k=k_{0}}^{\infty} 2^{-k(m(m+1)+p(\alpha-1))} .
\end{aligned}
$$

This sum is finite if and only if $\alpha>1-\frac{m(m+1)}{p}$.

\section{Construction of a $C^{1,1-2 / p}$ curve with infinite $\mathcal{M}_{p}$ energy}

In this section we shall prove the following theorem.

Theorem 5.1. Let $p>2$ and set $\alpha=1-\frac{2}{p}$. There exists a function $F \in C^{1, \alpha}(\mathbb{R})$ such that $\mathcal{M}_{p}\left(\operatorname{Graph}(F) \cap \mathbb{B}_{2}\right)=\infty$.

The construction of our function is based on the van der Waerden saw. Let

$$
\tilde{f}(x)=\left\{\begin{array}{ll}
2 x & \text { for } x \in\left[0, \frac{1}{2}\right] \\
2-2 x & \text { for } x \in\left(\frac{1}{2}, 1\right]
\end{array} \text { and } f_{0}(x)=\tilde{f}(x-[x]) .\right.
$$

Fix some $\alpha \in(0,1)$ and $N \in \mathbb{N}$ - the values of these constants shall be determined later on. For $k=1,2, \ldots$ we set

$$
f_{k}(x)=\frac{f_{0}\left(N^{k} x\right)}{N^{\alpha k}}, \quad f(x)=\sum_{k=0}^{\infty} f_{k}(x)
$$




$$
\text { and } F(x)=\int_{0}^{x} f(t) d t=\sum_{k=0}^{\infty} \int_{0}^{x} f_{k}(t) d t .
$$

First we show that $F$ is $C^{1, \alpha}$.

Lemma 5.2. The function $F: \mathbb{R} \rightarrow \mathbb{R}$ defined by (7) is of class $C^{1, \alpha}$ and we have

$$
\forall x, y \in \mathbb{R} \quad\left|F^{\prime}(x)-F^{\prime}(y)\right| \leq C(N, \alpha)|x-y|^{\alpha} .
$$

The proof can be considered as a simple exercise in calculus. Given two points $x, y \in \mathbb{R}$, to estimate $|f(x)-f(y)|$ one has to divide the sum $\sum f_{k}$ into two parts: $k<-\log _{N}|x-y|$ and $k \geq-\log _{N}|x-y|$. Then estimate each part separately using the fact that a finite sum of a geometric series with ratio $q>1$ is comparable to the last term in the sum and an infinite sum of a geometric series with ratio $q<1$ is comparable to the first term in the sum. Below we give the proof for the convenience of the reader.

Proof. Since $F^{\prime}(x)=f(x)$, it suffices to indicate that $f: \mathbb{R} \rightarrow \mathbb{R}$ is Hölder continuous. Note that $f$ is periodic with period 1, so it is enough to show that $|f(x)-f(y)| \lesssim|x-y|^{\alpha}$ for $x \in[0,1]$ and $y \in[0,1]$.

Fix two numbers $x \in[0,1]$ and $y \in[0,1]$ such that $x<y$. Let $h=y-x$ and let $l=\left\lceil-\log _{N} h\right\rceil$, so that $N^{-l} \leq|x-y| \leq N^{-l+1}$. We can express $x$ and $h$ as infinite sums

$$
x=\sum_{j=0}^{\infty} \frac{x_{j}}{N^{j}} \quad \text { and } \quad y-x=h=\sum_{j=l}^{\infty} \frac{h_{j}}{N^{j}},
$$

where $x_{j}, h_{j} \in\{0,1, \ldots, N-1\}$ for each $j \in \mathbb{N}$. For convenience we set $h_{0}=$ $\cdots=h_{l-1}=0$. Now, we calculate

$$
\begin{aligned}
|f(y)-f(x)| & =|f(x+h)-f(x)| \\
& =\left|\sum_{k=0}^{\infty} \frac{1}{N^{\alpha k}}\left(f_{0}\left(\sum_{j=0}^{\infty} \frac{N^{k} x_{j}}{N^{j}}\right)-f_{0}\left(\sum_{j=0}^{\infty} \frac{N^{k} x_{j}+N^{k} h_{j}}{N^{j}}\right)\right)\right| \\
& \leq \sum_{k=0}^{\infty} \frac{1}{N^{\alpha k}} \mid f_{0}\left(\sum_{j=\max (k+1, l)}^{\infty} \frac{N^{k} x_{j}}{N^{j}}\right)-f_{0}\left(\sum_{j=\max (k+1, l)}^{\infty} \frac{N^{k} x_{j}+N^{k} h_{j}}{N^{j}}\right) .
\end{aligned}
$$

Hence, using the fact that $f_{0}$ is Lipschitz continuous with Lipschitz constant 2 we obtain

$$
\begin{aligned}
|f(y)-f(x)| & \leq \sum_{k=0}^{l-1} \frac{2}{N^{\alpha k}} \sum_{j=l}^{\infty} \frac{N^{k} h_{j}}{N^{j}}+\sum_{k=l}^{\infty} \frac{2}{N^{\alpha k}} \sum_{j=k+1}^{\infty} \frac{N^{k} h_{j}}{N^{j}} \\
& \leq \frac{4}{N^{l}} \sum_{k=0}^{l-1} N^{k(1-\alpha)}+2 \sum_{k=l}^{\infty} \frac{N^{k}}{N^{\alpha k}} \sum_{j=k+1}^{\infty} \frac{N-1}{N^{j}} \\
& \leq \frac{4}{N^{\alpha l}}\left(\frac{N}{N^{1-\alpha}-1}+\frac{N^{\alpha}}{N^{\alpha}-1}\right) \leq C(N, \alpha)|y-x|^{\alpha} .
\end{aligned}
$$


Recall that $\alpha=1-\frac{2}{p}$. Now we shall prove that the $p$-integral curvature $\mathcal{M}_{p}\left(\operatorname{Graph}(F) \cap \mathbb{B}_{2}\right)$ is infinite.

Lemma 5.3. The function $F: \mathbb{R} \rightarrow \mathbb{R}$ defined by (7) satisfies $\mathcal{M}_{p}(\operatorname{Graph}(F) \cap$ $\left.\mathbb{B}_{2}\right)=\infty$.

Proof. The graph of $F$ is not a closed curve, thus we express the $\mathcal{M}_{p}$ energy in the following way

$$
\mathcal{M}_{p}\left(\operatorname{Graph}(F) \cap \mathbb{B}_{2}\right):=\int_{0}^{L} \int_{0}^{L} \int_{0}^{L} R^{-p}(\Gamma(x), \Gamma(y), \Gamma(z)) d x d y d z,
$$

where $\Gamma$ is an arc-length parameterization of $\operatorname{Graph}(F)$ and $L=\mathcal{H}^{1}(\operatorname{Graph}(F) \cap$ $\left.\mathbb{B}_{2}\right)$. It is easy to notice that

$\mathcal{M}_{p}\left(\operatorname{Graph}(F) \cap \mathbb{B}_{2}\right)>\int_{0}^{1} \int_{0}^{1} \int_{0}^{1} R^{-p}((x, F(x)),(y, F(y)),(z, F(z))) d x d y d z$

We denote by $\vec{t}$ a point of the graph given by argument $t$; i.e. $\vec{t}=(t, F(t))$. Since $F$ is a Lipschitz function, for any two points of the graph we have

$$
|t-s| \leq\|\vec{t}-\vec{s}\|=\sqrt{(t-s)^{2}+(F(t)-F(s))^{2}} \leq C(\alpha)|t-s| .
$$

Let us start with the following estimation of the Menger curvature of three points of the graph. If $0 \leq x \leq z \leq y \leq 1$ we have

$$
\begin{aligned}
\frac{1}{R(\vec{x}, \vec{y}, \vec{z})} & =\frac{4 \mathcal{H}^{2}(\Delta(\vec{x}, \vec{y}, \vec{z}))}{\|\vec{x}-\vec{y}\|\|\vec{y}-\vec{z}\|\|\vec{x}-\vec{z}\|} \geq \frac{2 h}{\|\vec{x}-\vec{y}\|^{2}} \\
& =\frac{2 \sin \Varangle(\vec{x}-\vec{y}, \vec{x}-\vec{z})}{\|\vec{x}-\vec{y}\|^{2}}\|\vec{x}-\vec{z}\|,
\end{aligned}
$$

where $h$ denotes the height of the triangle $\triangle(\vec{x}, \vec{y}, \vec{z})$ which is perpendicular to $\vec{x}-\vec{y}$. In order to find a lower bound for the above expression we estimate the tangent of the angle between $\vec{x}-\vec{y}$ and $\vec{x}-\vec{z}$. Using the mean value theorem and the fact that $F^{\prime}=f$ is nonnegative and bounded we obtain

$$
\begin{aligned}
|\tan (\Varangle(\vec{x}-\vec{y}, \vec{x}-\vec{z}))| & =\frac{\left|\frac{F(y)-F(x)}{y-x}-\frac{F(z)-F(x)}{z-x}\right|}{1+\frac{F(y)-F(x)}{y-x} \frac{F(z)-F(x)}{z-x}} \\
& \geq C(N, \alpha)\left|\frac{F(y)-F(x)}{y-x}-\frac{F(z)-F(x)}{z-x}\right| .
\end{aligned}
$$

Therefore if $\Varangle(\vec{x}-\vec{y}, \vec{x}-\vec{z}) \leq \frac{\pi}{3}$ we have

$$
\frac{1}{R(\vec{x}, \vec{y}, \vec{z})} \geq \frac{C(N, \alpha)\left|\frac{F(y)-F(x)}{y-x}-\frac{F(z)-F(x)}{z-x}\right|(z-x)}{(y-x)^{2}} .
$$


We will prove that the energy is infinite even when we consider a much smaller domain of integration. For $k \in \mathbb{N}$ and $m \in\left\{0,1, \ldots, N^{k}-1\right\}$ we define the following intervals

$$
\begin{aligned}
X_{k, m} & =\left[\frac{m}{N^{k}}, \frac{m}{N^{k}}+\frac{1}{16 N^{k}}\right], \\
Y_{k, m} & =\left[\frac{m+1 / 2}{N^{k}}-\frac{1}{16 N^{k}}, \frac{m+1 / 2}{N^{k}}\right], \\
Z_{k, m} & =\left[\frac{m+1 / 4}{N^{k}}-\frac{1}{16 N^{k}}, \frac{m+1 / 4}{N^{k}}+\frac{1}{16 N^{k}}\right] .
\end{aligned}
$$

It is easy to notice that the family $A_{k, m}=X_{k, m} \times Y_{k, m} \times Z_{k, m}$ indexed by $k, m \in \mathbb{N}$, $m<N^{k}$ is pairwise disjoint. Indeed if $k_{1}>k_{2}$ and $N$ is sufficiently large, then either $\left(X_{k_{1}, m_{1}} \cup Y_{k_{1}, m_{1}} \cup Z_{k_{1}, m_{1}}\right) \subset X_{k_{2}, m_{2}}$ (which implies $Y_{k_{1}, m_{1}} \cap Y_{k_{2}, m_{2}}=\emptyset$ ) or $\left(X_{k_{1}, m_{1}} \cup Y_{k_{1}, m_{1}} \cup Z_{k_{1}, m_{1}}\right) \cap X_{k_{2}, m_{2}}=\emptyset$. Thus

$$
\mathcal{M}_{p}\left(\operatorname{Graph}(F) \cap \mathbb{B}_{2}\right) \geq \sum_{k=1}^{\infty} \sum_{m=0}^{N^{k}-1} \int_{X_{k, m}} \int_{Y_{k, m}} \int_{Z_{k, m}} R^{-p}(\vec{x}, \vec{y}, \vec{z}) d x d y d z .
$$

Let us fix $k \in \mathbb{N}, m \in\left\{0,1, \ldots, N^{k}-1\right\}$ and $x \in X_{k, m}, y \in Y_{k, m}$ and $z \in Z_{k, m}$. Obviously if $N$ is sufficiently large, for such $x, y, z$ we have $\Varangle(\vec{x}-\vec{y}, \vec{x}-\vec{z}) \leq \frac{\pi}{3}$ and estimation (8) holds. Recall that we have

$$
F(x)=\sum_{j=0}^{\infty} \int_{0}^{x} f_{j}(t) d t .
$$

For convenience we denote $F_{j}(x)=\int_{0}^{x} f_{j}(t)$ and we notice that

$$
\left|\frac{F(y)-F(x)}{y-x}-\frac{F(z)-F(x)}{z-x}\right|=\left|\sum_{j=0}^{\infty} \frac{F_{j}(y)-F_{j}(x)}{y-x}-\frac{F_{j}(z)-F_{j}(x)}{z-x}\right| .
$$

We need to divide the above sum into two parts which behave differently.

- For $j \leq k$ there are two possibilities. Either

$$
\begin{aligned}
\frac{F_{j}(y)-F_{j}(x)}{y-x} & =\frac{1}{y-x} \int_{x}^{y}\left(\frac{1}{N^{j}}\right)^{\alpha} f_{0}\left(N^{j} t\right) d t \\
& =\frac{1}{y-x} \int_{x}^{y}\left(\frac{1}{N^{j}}\right)^{\alpha} 2 N^{j} t d t=\left(\frac{1}{N^{j}}\right)^{\alpha-1}(x+y)
\end{aligned}
$$

and

$$
\frac{F_{j}(z)-F_{j}(x)}{z-x}=\left(\frac{1}{N^{j}}\right)^{\alpha-1}(x+z)
$$


or

$$
\begin{aligned}
\frac{F_{j}(y)-F_{j}(x)}{y-x} & =\frac{1}{y-x} \int_{x}^{y}\left(\frac{1}{N^{j}}\right)^{\alpha} 2\left(1-N^{j} t\right) d t \\
& =\left(\frac{1}{N^{j}}\right)^{\alpha}-\left(\frac{1}{N^{j}}\right)^{\alpha-1}(x+y)
\end{aligned}
$$

and

$$
\frac{F_{j}(z)-F_{j}(x)}{z-x}=\left(\frac{1}{N^{j}}\right)^{\alpha}-\left(\frac{1}{N^{j}}\right)^{\alpha-1}(x+z) .
$$

Denoting by

$$
\delta_{j}(x, z, y):=\frac{F_{j}(y)-F_{j}(x)}{y-x}-\frac{F_{j}(z)-F_{j}(x)}{z-x},
$$

in both cases we have

$$
\left|\delta_{j}(x, z, y)\right|=\left(N^{j}\right)^{1-\alpha}(y-z) .
$$

Now, taking $N$ sufficiently large:

$$
\left|\sum_{j=1}^{k} \delta_{j}(x, z, y)\right| \geq \frac{1}{2}\left(N^{k}\right)^{1-\alpha}(y-z) \geq \frac{1}{2}\left(N^{k}\right)^{1-\alpha} \frac{3}{16} \frac{1}{N^{k}}=\frac{3}{32} N^{-k \alpha} \text {. (9) }
$$

- For $j>k$ we notice that

$$
\begin{aligned}
F_{j}(y)-F_{j}(x) & =\int_{x}^{y} f_{j}(t) d t \leq\left\lfloor(y-x) 2 N^{j}\right\rfloor \frac{1}{4 N^{j}}\left(\frac{1}{N^{j}}\right)^{\alpha}+\frac{1}{2 N^{j}}\left(\frac{1}{N^{j}}\right)^{\alpha} \\
& \leq(y-x) \frac{1}{2}\left(\frac{1}{N^{j}}\right)^{\alpha}+\frac{1}{2}\left(\frac{1}{N^{j}}\right)^{\alpha+1}
\end{aligned}
$$

and

$$
F_{j}(y)-F_{j}(x) \geq \frac{1}{2}(y-x)\left(\frac{1}{N^{j}}\right)^{\alpha}-\frac{1}{4}\left(\frac{1}{N^{j}}\right)^{\alpha+1} .
$$

As $y-x \geq \frac{3}{8} \frac{1}{N^{k}}$ we have

$$
\begin{aligned}
\frac{1}{2}\left(\frac{1}{N^{j}}\right)^{\alpha}-\frac{2}{3} N^{k}\left(\frac{1}{N^{j}}\right)^{\alpha+1} & \leq \frac{F_{j}(y)-F_{j}(x)}{y-x} \\
& \leq \frac{1}{2}\left(\frac{1}{N^{j}}\right)^{\alpha}+\frac{4}{3} N^{k}\left(\frac{1}{N^{j}}\right)^{\alpha+1}
\end{aligned}
$$


Analogously, since $z-x \geq \frac{1}{8} \frac{1}{N^{k}}$

$$
\begin{aligned}
\frac{1}{2}\left(\frac{1}{N^{j}}\right)^{\alpha}-2 N^{k}\left(\frac{1}{N^{j}}\right)^{\alpha+1} & \leq \frac{F_{j}(z)-F_{j}(x)}{z-x} \\
& \leq \frac{1}{2}\left(\frac{1}{N^{j}}\right)^{\alpha}+4 N^{k}\left(\frac{1}{N^{j}}\right)^{\alpha+1} .
\end{aligned}
$$

Thus

$$
\left|\delta_{j}(x, z, y)\right|<5 N^{k}\left(\frac{1}{N^{j}}\right)^{\alpha+1} .
$$

We can choose $N$ large enough to estimate the geometric series from above by its first term

$$
\left|\sum_{j=k+1}^{\infty} \delta_{j}(x, z, y)\right|<\sum_{j=k+1}^{\infty} 5 N^{k}\left(\frac{1}{N^{j}}\right)^{\alpha+1}<10 N^{k}\left(\frac{1}{N^{k+1}}\right)^{\alpha+1},
$$

enlarging $N$ if necessary we get

$$
\left|\sum_{j=k+1}^{\infty} \delta_{j}(x, z, y)\right|<\frac{1}{32}\left(\frac{1}{N^{k}}\right)^{\alpha} .
$$

Putting both estimates (9) and (10) together we obtain

$$
\begin{aligned}
\left|\sum_{j=0}^{\infty} \delta_{j}(x, z, y)\right| & \geq\left|\sum_{j=0}^{k} \delta_{j}(x, z, y)\right|-\left|\sum_{j=k+1}^{\infty} \delta_{j}(x, z, y)\right| \\
& \geq \frac{3}{32}\left(\frac{1}{N^{k}}\right)^{\alpha}-\frac{1}{32}\left(\frac{1}{N^{k}}\right)^{\alpha}=\frac{1}{16}\left(\frac{1}{N^{k}}\right)^{\alpha} .
\end{aligned}
$$

Thus for each $k \in \mathbb{N}, m \in\left\{1,2, \ldots, N^{k}-1\right\}$ and $x \in X_{k, m}, y \in Y_{k, m}, z \in Z_{k, m}$ we have

$$
\left|\frac{F(y)-F(x)}{y-x}-\frac{F(z)-F(x)}{z-x}\right| \geq \frac{1}{16}\left(\frac{1}{N^{k}}\right)^{\alpha} .
$$

Using (11) we can estimate the integral

$$
\begin{aligned}
& \mathcal{M}_{p}\left(\operatorname{Graph}(F) \cap \mathbb{B}_{2}\right) \\
& \geq \sum_{k=1}^{\infty} \sum_{m=0}^{N^{k}-1} \int_{X_{k, m}} \int_{Y_{k, m}} \int_{Z_{k, m}} R^{-p}(\vec{x}, \vec{y}, \vec{z}) d z d y d x \\
& \geq \sum_{k=1}^{\infty} \sum_{m=0}^{N^{k}-1} \int_{X_{k, m}} \int_{Y_{k, m}} \int_{Z_{k, m}} C(\alpha)\left(\frac{\frac{1}{16} N^{-k \alpha}(z-x)}{(y-x)^{2}}\right)^{p} d z d y d x
\end{aligned}
$$




$$
\begin{aligned}
& \geq C(\alpha) \sum_{k=1}^{\infty} \sum_{m=0}^{N^{k}-1}\left(\frac{\frac{1}{16} N^{-k \alpha} \frac{1}{8} N^{-k}}{\frac{1}{4} N^{-2 k}}\right)^{p}\left|X_{k, m}\right| \cdot\left|Y_{k, m}\right| \cdot\left|Z_{k, m}\right| \\
& \geq C(N, \alpha) \sum_{k=1}^{\infty} \sum_{m=0}^{N^{k}-1}\left(N^{k}\right)^{(1-\alpha) p-3} \geq C(N, \alpha) \sum_{k=1}^{\infty}\left(N^{k}\right)^{p-p \alpha-2} .
\end{aligned}
$$

Hence, the energy of the graph of $F$ is infinite whenever

$$
p-p \alpha-2 \geq 0 \quad \alpha \quad \alpha \leq 1-\frac{2}{p} \text {. }
$$

\section{Higher dimensional case}

Here we establish an analogue of Theorem 5.1 for the energy $\mathcal{E}_{p}$.

Theorem 6.1. Let $p>m(m+1)$ and set $\alpha=1-\frac{m(m+1)}{p}$. There exists a compact manifold $\Sigma$ of class $C^{1, \alpha}$ such that $\mathcal{E}_{p}(\Sigma)$ is infinite.

Our construction is based on the same idea as the construction presented in Sect. 5. Let $N \in \mathbb{N}$ be a big natural number and let $F: \mathbb{R} \rightarrow \mathbb{R}$ be defined by (7). We define

$$
G: \mathbb{R}^{m} \rightarrow \mathbb{R}^{m+1} \text { by the formula } G\left(x^{1}, \ldots, x^{m}\right)=\left(x^{1}, \ldots, x^{m}, F\left(x^{1}\right)\right)
$$

and we set $\Sigma=G\left([0,1]^{m}\right)$. From Lemma 5.2 it follows that $\Sigma$ is a $C^{1, \alpha}$ manifold.

To perform the proof of Theorem 6.1 we need to introduce some additional notation. By $\left(\mathbf{e}_{1}, \ldots, \mathbf{e}_{m}\right)$ we denote the standard basis of $\mathbb{R}^{m}$. We adopt the convention to typeset points and vectors in $\mathbb{R}^{m}$ with the bold font $\mathbf{x}, \mathbf{y}, \mathbf{z}$ etc. and to number the components with a superscript, so we shall have $\mathbf{x}=\left(x^{1}, \ldots, x^{m}\right)$. Also, vectors and points in $\mathbb{R}^{m+1}$ will always be marked with an arrow $\overrightarrow{\mathbf{x}}, \overrightarrow{\mathbf{y}}, \overrightarrow{\mathbf{z}}$ etc. and we silently assume that $\mathbf{x}$ and $\overrightarrow{\mathbf{x}}$ always satisfy

$$
\pi_{\mathbb{R}^{m}}(\overrightarrow{\mathbf{x}})=\pi_{\mathbb{R}^{m}}\left(x^{1}, \ldots, x^{m+1}\right)=\left(x^{1}, \ldots, x^{m}\right)=\mathbf{x} .
$$

Let $U$ and $V$ be any $m$-dimensional subspaces of $\mathbb{R}^{m+1}$. We define

$$
Q_{U}:=\pi_{U^{\perp}}=\mathrm{id}-\pi_{U} \text { and } \Varangle(U, V):=\left\|\pi_{U}-\pi_{V}\right\|=\left\|Q_{U}-Q_{V}\right\| .
$$

For $\mathbf{z} \in \mathbb{R}^{m}$ and $r>0$ we also write

$$
\begin{aligned}
& \mathbb{B}_{+}^{m}(\mathbf{z}, r)=\mathbb{B}^{m}(\mathbf{z}, r) \cap\left(\left(z^{1}, \infty\right) \times \mathbb{R}^{m-1}\right) \text { and } \\
& \mathbb{B}_{-}^{m}(\mathbf{z}, r)=\mathbb{B}^{m}(\mathbf{z}, r) \cap\left(\left(-\infty, z^{1}\right) \times \mathbb{R}^{m-1}\right) .
\end{aligned}
$$

Let $\varepsilon>0$ be a small constant - its value will be fixed later on depending only on $m$. Let $A=A(N, \alpha, \varepsilon) \in(0,1)$ be such that $\left|F^{\prime}(t)\right| \leq \varepsilon$ whenever $t \leq A$. For $n \in \mathbb{N}$ we set

$$
\begin{aligned}
J_{n} & =N^{-n} \mathbb{Z}^{m} \cap[0, A)^{m} \\
& =\left\{\left(\frac{k_{1}}{N^{n}}, \ldots, \frac{k_{m}}{N^{n}}\right): \forall i \in\{1, \ldots, m\} k_{i} \in\left\{0,1, \ldots,\left\lceil A N^{n}-1\right\rceil\right\}\right\} .
\end{aligned}
$$


For any $\mathbf{x} \in J_{n}$ we define

$$
\mathbf{x}_{0}^{(\mathbf{x})}=\mathbf{x}, \quad \mathbf{x}_{k}^{(\mathbf{x})}=\mathbf{x}+\frac{\mathbf{e}_{k}}{2 N^{n}} \quad \text { for } k=1, \ldots, m \quad \text { and } \quad \mathbf{x}_{m+1}^{(\mathbf{x})}=\mathbf{x}+\frac{\mathbf{e}_{1}}{4 N^{n}} .
$$

Of course $\mathbf{x}_{i}^{(\mathbf{x})}$ depends on $n$ but we do not highlight it in our notation. For $\delta \in\left(0, \frac{1}{32}\right)$ we set

$U_{n}^{\delta}(\mathbf{x})=\mathbb{B}_{+}^{m}\left(\mathbf{x}_{0}^{(\mathbf{x})}, \frac{\delta}{N^{n}}\right) \times \mathbb{B}_{-}^{m}\left(\mathbf{x}_{1}^{(\mathbf{x})}, \frac{\delta}{N^{n}}\right) \times \mathbb{B}^{m}\left(\mathbf{x}_{2}^{(\mathbf{x})}, \frac{\delta}{N^{n}}\right) \times \cdots \times \mathbb{B}^{m}\left(\mathbf{x}_{m+1}^{(\mathbf{x})}, \frac{\delta}{N^{n}}\right)$.

Note that

$$
\mathcal{H}^{0}\left(J_{n}\right)=\left\lceil A N^{n}\right\rceil^{m} \geq A^{m} N^{n m},
$$

$\forall n_{1}, n_{2} \in \mathbb{N} \forall \mathbf{x} \in J_{n_{1}} \forall \mathbf{y} \in J_{n_{2}} \quad\left(\mathbf{x} \neq \mathbf{y} \vee n_{1} \neq n_{2}\right) \Rightarrow U_{n_{1}}^{\delta}(\mathbf{x}) \cap U_{n_{2}}^{\delta}(\mathbf{y})=\emptyset$

$$
\text { and } \forall \mathbf{x} \in J_{n} \quad \mathcal{H}^{m(m+2)}\left(U_{n}^{\delta}(\mathbf{x})\right)=\frac{1}{4}\left(\frac{\omega_{m} \delta^{m}}{N^{n m}}\right)^{m+2} \text {. }
$$

Let $T=\left(\mathbf{z}_{0}, \ldots, \mathbf{z}_{m+1}\right) \in\left(\mathbb{R}^{m}\right)^{m+2}$ and $G(T)=\left(G\left(\mathbf{z}_{0}\right), \ldots, G\left(\mathbf{z}_{m+1}\right)\right)=$ $\left(\overrightarrow{\mathbf{z}}_{0}, \ldots, \overrightarrow{\mathbf{z}}_{m+1}\right)$. We define

$\mathfrak{f} \mathfrak{c}(T)=\triangle\left\{\overrightarrow{\mathbf{z}}_{0}, \ldots, \overrightarrow{\mathbf{z}}_{m}\right\}$ - the face of $G(T)$ spanned by $\overrightarrow{\mathbf{z}}_{0}, \ldots, \overrightarrow{\mathbf{z}}_{m}$,

$\mathfrak{p}(T)=\operatorname{span}\left\{\overrightarrow{\mathbf{z}}_{1}-\overrightarrow{\mathbf{z}}_{0}, \ldots, \overrightarrow{\mathbf{z}}_{m}-\overrightarrow{\mathbf{z}}_{0}\right\}$ - the vector space containing $\mathfrak{f} \mathfrak{c}(T)-\overrightarrow{\mathbf{z}}_{0}$

and $\mathfrak{h}(T)=\operatorname{dist}\left(\overrightarrow{\mathbf{z}}_{m+1}, \overrightarrow{\mathbf{z}}_{0}+\mathfrak{p}(T)\right)$ - the height of $G(T)$ lowered from $\overrightarrow{\mathbf{z}}_{m+1}$.

For brevity we also write

$$
\mathcal{K}_{G}(T)=\mathcal{K}(G(T))=\mathcal{K}\left(G\left(\mathbf{z}_{0}\right), \ldots, G\left(\mathbf{z}_{m+1}\right)\right)
$$

Note that $|J G(\mathbf{x})|=\sqrt{\operatorname{det}\left(D G(\mathbf{x})^{T} D G(\mathbf{x})\right)} \geq 1$, so we have

$$
\mathcal{E}_{p}(\Sigma) \geq \int_{[0,1]^{m(m+2)}} \mathcal{K}_{G}\left(\mathbf{z}_{0}, \ldots, \mathbf{z}_{m+1}\right)^{p} d \mathbf{z}_{0} \cdots d \mathbf{z}_{m+1}
$$

Proposition 6.2. There exists $\delta_{0}=\delta_{0}(m, N, \alpha) \in\left(0, \frac{1}{32}\right)$ such that for all $n \in \mathbb{N}$ and for any $\delta \leq \delta_{0}$, if $\mathbf{x} \in J_{n}$ and $T=\left(\mathbf{z}_{0}, \ldots, \mathbf{z}_{m+1}\right) \in U_{n}^{\delta}(\mathbf{x})$, then we have

$$
\mathfrak{h}(T) \geq \frac{1}{320 N^{n(1+\alpha)}} \text { and } \mathcal{K}_{G}(T) \geq C(\delta, m) N^{n(1-\alpha)} .
$$

Using Proposition 6.2 we can finish the proof of the main theorem. 
Proof of Theorem. 6.1. Using (12), (13) and (14) together with (15) we get

$$
\begin{aligned}
\mathcal{E}_{p}(\Sigma) & \geq \sum_{n=1}^{\infty} \sum_{\mathbf{x} \in J_{n}} \int_{U_{n}^{\delta}(\mathbf{x})} \mathcal{K}_{G}(T)^{p} d \mathbf{z}_{0} \cdots d \mathbf{z}_{m+1} \\
& \geq C(\delta, m, p) \sum_{n=1}^{\infty} A^{m} N^{n m} \frac{\omega_{m}^{m+2} \delta^{m(m+2)}}{4 N^{n m(m+2)}} N^{n(1-\alpha) p} \\
& =\hat{C}(\delta, m, p, N, \alpha, \varepsilon) \sum_{n=1}^{\infty} N^{n(m-m(m+2)+(1-\alpha) p)},
\end{aligned}
$$

which is infinite if and only if $\alpha \leq 1-\frac{m(m+1)}{p}$. In particular, for $\alpha=1-\frac{m(m+1)}{p}$ we have $\mathcal{E}_{p}(\Sigma)=\infty$.

Now we only need to prove Proposition 6.2.

Proof of Theorem. 6.2. Recall that $T=\left(\mathbf{z}_{0}, \ldots, \mathbf{z}_{m+1}\right) \in U_{n}^{\delta}(\mathbf{x})$ and $\mathbf{x} \in J_{n}$, so $T$ and $\mathbf{x}$ depend on $n$. Note that the vectors $\mathbf{z}_{i}-\mathbf{z}_{0}$ for $i, j=1, \ldots, m$ and $i \neq j$ satisfy

$$
\begin{aligned}
& (1-4 \delta) \frac{1}{2 N^{n}} \leq\left|\mathbf{z}_{i}-\mathbf{z}_{0}\right| \leq(1+4 \delta) \frac{1}{2 N^{n}} \\
& \text { and }\left|\left\langle\mathbf{z}_{i}-\mathbf{z}_{0}, \mathbf{z}_{j}-\mathbf{z}_{0}\right\rangle\right| \leq \frac{3 \delta}{N^{2 n}}
\end{aligned}
$$

Hence, the vectors $\mathbf{z}_{i}-\mathbf{z}_{0}$ for $i=1, \ldots, m$ form a roughly orthogonal basis of $\mathbb{R}^{m}$. Recall that $\left|F^{\prime}(t)\right| \leq \varepsilon$ for all $t \in[0, A]$. We can find $\delta_{1}=\delta_{1}(m)>0$ and $\varepsilon=\varepsilon(m)>0$ such that for any $\delta \leq \delta_{1}$ and for each $n \in \mathbb{N}$ (see [7, Proposition 1.23] for the proof)

$$
\Varangle\left(\mathfrak{p}(T), \mathbb{R}^{m}\right)=\left\|\pi_{\mathfrak{p}(T)}-\pi_{\mathbb{R}^{m}}\right\|=\left\|Q_{\mathfrak{p}(T)}-Q_{\mathbb{R}^{m}}\right\| \leq \frac{1}{2} .
$$

Since the vector $\mathbf{z}_{m+1}-\mathbf{z}_{0}$ lies in $\mathbb{R}^{m}$, it can be expressed as

$$
\mathbf{z}_{m+1}-\mathbf{z}_{0}=\sum_{i=1}^{m} \zeta_{i}\left(\mathbf{z}_{i}-\mathbf{z}_{0}\right),
$$

for some $\zeta_{1}, \ldots, \zeta_{m}$. Observe that when we decrease $\delta$ to zero, the point $\mathbf{z}_{m+1}$ approaches the midpoint $\frac{1}{2}\left(\mathbf{z}_{0}+\mathbf{z}_{1}\right)$, so $\zeta_{1}$ converges to $\frac{1}{2}$ and all the other $\zeta_{i}$ for $i=2, \ldots, m$ converge to 0 . The values $\left|\zeta_{1}-\frac{1}{2}\right|$ and $\left|\zeta_{i}\right|$ can be bounded above independently of the scale we are working in (i.e. independently of the choice of $n$ ) and also independently of the choice of $T$ in $U_{n}^{\delta}(\mathbf{x})$. Hence

$$
\left|\zeta_{1}-\frac{1}{2}\right| \leq \Xi(\delta) \text { and }\left|\zeta_{i}\right| \leq \Xi(\delta) \text { for } i=2, \ldots, m, \text { where } \Xi(\delta) \stackrel{\delta \rightarrow 0}{\longrightarrow} 0
$$


and $\Xi(\delta)$ is a term depending only on $\delta$ and $m$. Therefore, we can find $\delta_{2}=\delta_{2}(m) \in$ $\left(0, \delta_{1}\right)$ such that for each $\delta \leq \delta_{2}$ the following holds $\Xi(\delta)<\frac{1}{32 m}$. We shall always assume that $\delta \leq \delta_{2}$ in the sequel.

For $i=1, \ldots, m+1$ we set

$$
\overrightarrow{\mathbf{z}}_{i}=G\left(\mathbf{z}_{i}\right) \quad \text { and we define } \quad \overrightarrow{\mathbf{w}}=\overrightarrow{\mathbf{z}}_{0}+\sum_{i=1}^{m} \zeta_{i}\left(\overrightarrow{\mathbf{z}}_{i}-\overrightarrow{\mathbf{z}}_{0}\right) \in \overrightarrow{\mathbf{z}}_{0}+\mathfrak{p}(T)
$$

Then $\mathbf{z}_{m+1}=\pi_{\mathbb{R}^{m}}\left(\overrightarrow{\mathbf{z}}_{m+1}\right)=\pi_{\mathbb{R}^{m}}(\overrightarrow{\mathbf{w}})$ and $\left(\overrightarrow{\mathbf{z}}_{m+1}-\overrightarrow{\mathbf{w}}\right) \perp \mathbb{R}^{m}$ and also $Q_{\mathfrak{p}(T)}\left(\overrightarrow{\mathbf{z}}_{m+1}-\right.$ $\overrightarrow{\mathbf{w}})=Q_{\mathfrak{p}(T)}\left(\overrightarrow{\mathbf{z}}_{m+1}-\overrightarrow{\mathbf{z}}_{0}\right)$. Therefore, using the angle estimate (16) we obtain

$$
\begin{aligned}
\mathfrak{h}(T) & =\operatorname{dist}\left(\overrightarrow{\mathbf{z}}_{m+1}, \overrightarrow{\mathbf{z}}_{0}+\mathfrak{p}(T)\right)=\left|Q_{\mathfrak{p}(T)}\left(\overrightarrow{\mathbf{z}}_{m+1}-\overrightarrow{\mathbf{z}}_{0}\right)\right| \\
& =\left|Q_{\mathfrak{p}(T)}\left(\overrightarrow{\mathbf{z}}_{m+1}-\overrightarrow{\mathbf{w}}\right)\right|=\left|Q_{\mathbb{R}^{m}}\left(\overrightarrow{\mathbf{z}}_{m+1}-\overrightarrow{\mathbf{w}}\right)-\left(Q_{\mathbb{R}^{m}}-Q_{\mathfrak{p}(T)}\right)\left(\overrightarrow{\mathbf{z}}_{m+1}-\overrightarrow{\mathbf{w}}\right)\right| \\
& \geq\left|\overrightarrow{\mathbf{z}}_{m+1}-\overrightarrow{\mathbf{w}}\right|-\frac{1}{2}\left|\overrightarrow{\mathbf{z}}_{m+1}-\overrightarrow{\mathbf{w}}\right|=\frac{1}{2}\left|\overrightarrow{\mathbf{z}}_{m+1}-\overrightarrow{\mathbf{w}}\right| .
\end{aligned}
$$

Hence, to get a lower bound on $\mathfrak{h}(T)$ it suffices to estimate $\left|\overrightarrow{\mathbf{z}}_{m+1}-\overrightarrow{\mathbf{w}}\right|$ from below. We calculate

$$
\begin{aligned}
\left|\overrightarrow{\mathbf{z}}_{m+1}-\overrightarrow{\mathbf{w}}\right| & \left|\left(\mathbf{z}_{m+1}, F\left(z_{m+1}^{1}\right)\right)-\left(\mathbf{z}_{m+1}, F\left(z_{0}^{1}\right)+\sum_{i=1}^{m} \zeta_{i}\left(F\left(z_{i}^{1}\right)-F\left(z_{0}^{1}\right)\right)\right)\right| \\
= & \left|F\left(z_{m+1}^{1}\right)-F\left(z_{0}^{1}\right)-\sum_{i=1}^{m} \zeta_{i}\left(F\left(z_{i}^{1}\right)-F\left(z_{0}^{1}\right)\right)\right| \\
\geq & \left|F\left(z_{0}^{1}+\zeta_{1}\left(z_{1}^{1}-z_{0}^{1}\right)\right)-F\left(z_{0}^{1}\right)-\zeta_{1}\left(F\left(z_{1}^{1}\right)-F\left(z_{0}^{1}\right)\right)\right| \\
& -\mid\left(F\left(z_{0}^{1}+\zeta_{1}\left(z_{1}^{1}-z_{0}^{1}\right)+\sum_{i=2}^{m} \zeta_{i}\left(z_{i}^{1}-z_{0}^{1}\right)\right)-F\left(z_{0}^{1}+\zeta_{1}\left(z_{1}^{1}-z_{0}^{1}\right)\right)\right) \\
& -\sum_{i=2}^{m} \zeta_{i}\left(F\left(z_{i}^{1}\right)-F\left(z_{0}^{1}\right)\right) \mid .
\end{aligned}
$$

Observe that, since $\delta<\frac{1}{32}$, there exists an $l \in\left\{0,1, \ldots, N^{n}-1\right\}$ such that $z_{0}^{1} \in$ $X_{n, l}, z_{1}^{1} \in Y_{n, l}$. Moreover, since $\Xi(\delta)<\frac{1}{32}$ we also have $z_{0}^{1}+\zeta_{1}\left(z_{1}^{1}-z_{0}^{1}\right) \in Z_{n, l}$. Using the estimate $\zeta_{1}\left(z_{1}^{1}-z_{0}^{1}\right) \geq \frac{1}{5 N^{n}}$ and applying (11) we obtain ${ }^{2}$

$$
\left|F\left(z_{0}^{1}+\zeta_{1}\left(z_{1}^{1}-z_{0}^{1}\right)\right)-F\left(z_{0}^{1}\right)-\zeta_{1}\left(F\left(z_{1}^{1}\right)-F\left(z_{0}^{1}\right)\right)\right| \geq \frac{1}{80 N^{n(1+\alpha)}} .
$$

\footnotetext{
${ }^{2}$ Here $z_{0}^{1}, z_{1}^{1}$ and $z_{0}^{1}+\zeta_{1}\left(z_{1}^{1}-z_{0}^{1}\right)$ play the roles of $x, y$ and $z$ from (11) and $\frac{z-x}{y-x}=\zeta_{1}$.
} 
We estimate the remaining terms using the mean value theorem for $F$. First we have

$$
\begin{aligned}
& F\left(z_{0}^{1}+\zeta_{1}\left(z_{1}^{1}-z_{0}^{1}\right)+\sum_{i=2}^{m} \zeta_{i}\left(z_{i}^{1}-z_{0}^{1}\right)\right)-F\left(z_{0}^{1}+\zeta_{1}\left(z_{1}^{1}-z_{0}^{1}\right)\right) \\
& \quad=F^{\prime}\left(\xi_{m+1}\right) \sum_{i=2}^{m} \zeta_{i}\left(z_{i}^{1}-z_{0}^{1}\right),
\end{aligned}
$$

where $\xi_{m+1}$ lies between $z_{0}^{1}+\zeta_{1}\left(z_{1}^{1}-z_{0}^{1}\right)$ and $z_{0}^{1}+\zeta_{1}\left(z_{1}^{1}-z_{0}^{1}\right)+\sum_{i=2}^{m} \zeta_{i}\left(z_{i}^{1}-z_{0}^{1}\right)$. Recall that for $\delta \leq \delta_{2}$ we have $\Xi(\delta)<\frac{1}{32 m}$, so for such $\delta$ we easily get the estimate

$$
\left|\xi_{m+1}-\left(x^{1}+\frac{1}{4 N^{n}}\right)\right|<\frac{1}{2 N^{n}} .
$$

In the same way we obtain

$$
\sum_{i=2}^{m} \zeta_{i}\left(F\left(z_{i}^{1}\right)-F\left(z_{0}^{1}\right)\right)=\sum_{i=2}^{m} F^{\prime}\left(\xi_{i}\right) \zeta_{i}\left(z_{i}^{1}-z_{0}^{1}\right),
$$

where $\xi_{i}$ lies between $z_{0}^{1}$ and $z_{i}^{1}$, so $\left|\xi_{i}-x^{1}\right|<\frac{1}{32 N^{n}}$. Note that for $i=2, \ldots, m$ we have

$$
\left|\xi_{m+1}-\xi_{i}\right| \leq \frac{1}{N^{n}}, \quad\left|z_{i}^{1}-z_{0}^{1}\right| \leq \frac{2 \delta}{N^{n}}
$$

and

$$
\left|F^{\prime}\left(\xi_{i}\right)-F^{\prime}\left(\xi_{m+1}\right)\right| \leq C(N, \alpha)\left|\xi_{i}-\xi_{m+1}\right|^{\alpha} \leq C(N, \alpha) N^{-n \alpha} .
$$

Putting (21) and (22) together we get

$$
\begin{aligned}
& \mid\left(F\left(z_{0}^{1}+\zeta_{1}\left(z_{1}^{1}-z_{0}^{1}\right)+\sum_{i=2}^{m} \zeta_{i}\left(z_{i}^{1}-z_{0}^{1}\right)\right)-F\left(z_{0}^{1}+\zeta_{1}\left(z_{1}^{1}-z_{0}^{1}\right)\right)\right) \\
& \quad-\sum_{i=2}^{m} \zeta_{i}\left(F\left(z_{i}^{1}\right)-F\left(z_{0}^{1}\right)\right)|=| \sum_{i=2}^{m}\left(F^{\prime}\left(\xi_{i}\right)-F^{\prime}\left(\xi_{m+1}\right)\right) \zeta_{i}\left(z_{i}^{1}-z_{0}^{1}\right) \mid \\
& \quad \leq \frac{\check{C}(N, \alpha) \delta}{N^{n(1+\alpha)}} .
\end{aligned}
$$

Plugging (20) and (23) into (19) and recalling (18) we obtain

$$
\mathfrak{h}(T) \geq \frac{1}{2}\left|\overrightarrow{\mathbf{z}}_{m+1}-\overrightarrow{\mathbf{w}}\right| \geq \frac{\frac{1}{80}-\check{C}(N, \alpha) \delta}{2 N^{n(1+\alpha)}} .
$$

Setting $\delta_{0}=\min \left\{\delta_{1}, \delta_{2},(160 \check{C}(N, \alpha))^{-1}\right\}$ the first part of Proposition 6.2 is proven. The second part follows from a simple calculation

$$
\begin{aligned}
\mathcal{K}_{G}(T) & =\frac{\mathcal{H}^{m+1}(\triangle(G(T)))}{\operatorname{diam}(G(T))^{m+2}}=\frac{\mathfrak{h}(T) \mathcal{H}^{m}(\mathfrak{f} \mathfrak{c}(T))}{(m+1) \operatorname{diam}(G(T))^{m+2}} \\
& \geq \frac{\mathfrak{h}(T) \mathcal{H}^{m}\left(\pi_{\mathbb{R}^{m}}(\mathfrak{f} \mathfrak{c}(T))\right)}{(m+1) \operatorname{diam}(T)^{m+2}} \geq C(m, \delta) \frac{N^{-n(1+\alpha)} N^{-n m}}{N^{-n(m+2)}}=C(m, \delta) N^{n(1-\alpha)} .
\end{aligned}
$$


Acknowledgments. The first author was partially supported by the Polish Ministry of Science grant no. N N201 611140 (years 2011-2012). The second author was partially supported by the joint German-Polish project "Geometric curvature energies". The authors are indebted to Prof. P. Strzelecki for his valuable suggestions. The second author would like to thank Jonas Azzam for his questions, ideas and fruitful discussions.

Open Access This article is distributed under the terms of the Creative Commons Attribution Noncommercial License which permits any noncommercial use, distribution, and reproduction in any medium, provided the original author(s) and source are credited.

\section{References}

[1] Banavar, J.R., Gonzalez, O., Maddocks, J.H., Maritan, A.: Self-interactions of strands and sheets. J. Stat. Phys. 110(1-2), 35-50 (2003)

[2] Blatt, S.: A note on integral Menger curvature for curves. Preprint (2011)

[3] Blatt, S., Kolasiński, S.: Sharp boundedness and regularizing effects of the integral Menger curvature for submanifolds. Adv. Math. 230(3):839-852 (2012). doi:10.1016/ j.aim.2012.03.007.

[4] David, G., Semmes, S.: Analysis of and on Uniformly Rectifiable Sets, vol. 38 of Mathematical Surveys and Monographs. American Mathematical Society, Providence (1993)

[5] Gonzalez, O., Maddocks, J.H.: Global curvature, thickness, and the ideal shapes of knots. Proc. Natl Acad. Sci. USA 96(9), 4769-4773 (1999)

[6] Gonzalez, O., Maddocks, J.H., Schuricht, F., von der Mosel, H.: Global curvature and self-contact of nonlinearly elastic curves and rods. Calc. Var. Partial Differ. Equ. 14(1), 29-68 (2002)

[7] Kolasiński, S.: Integral Menger curvature for sets of arbitrary dimension and codimension. PhD thesis, Institute of Mathematics, University of Warsaw (2011). arXiv:1011:2008

[8] Léger, J.C.: Menger curvature and rectifiability. Ann. Math. (2) 149(3), 831-869 (1999)

[9] Lerman, G., Whitehouse, J.T.: High-dimensional Menger-type curvatures. II. $d$-separation and a menagerie of curvatures. Constr. Approx. 30(3), 325-360 (2009)

[10] Lerman, G., Whitehouse, J.T.: High-dimensional Menger-type curvatures. Part I: Geometric multipoles and multiscale inequalities. Rev. Mat. Iberoam. 27(2), 493-555 (2011)

[11] Mattila, P.: Rectifiability, analytic capacity, and singular integrals. In: Proceedings of the International Congress of Mathematicians, vol. II (Berlin, 1998), number Extra vol. II, pp. 657-664 (electronic), 1998

[12] Melnikov, M.S.: Analytic capacity: a discrete approach and the curvature of measure. Mat. Sb. 186(6), 57-76 (1995)

[13] Pajot, H.: Analytic Capacity, Rectifiability, Menger Curvature and the Cauchy Integral, vol. 1799 of Lecture Notes in Mathematics. Springer, Berlin (2002)

[14] Strzelecki, P., von der Mosel, H.: On rectifiable curves with $l^{p}$-bounds on global curvature: self-avoidance, regularity, and minimizing knots. Math. Z. 257(1), 107-130 (2007)

[15] Strzelecki, P., von der Mosel, H.: Integral Menger curvature for surfaces. Adv. Math. 226(3), 2233-2304 (2011)

[16] Strzelecki, P., von der Mosel, H.: Tangent-point repulsive potentials for a class of non-smooth $m$-dimensional sets in $\mathbb{R}^{n}$. Part I: smoothing and self-avoidance effects. J. Geom. Anal. (2011). doi:10.1007/s12220-011-9275-z. 
[17] Strzelecki, P., von der Mosel, H.: Tangent-point self-avoidance energies for curves. J. Knot Theory Ramifications 21(5), 28 pp (2012)

[18] Strzelecki, P., Szumańska, M., von der Mosel, H.: A geometric curvature double integral of Menger type for space curves. Ann. Acad. Sci. Fenn. Math. 34(1), 195-214 (2009)

[19] Strzelecki, P., Szumańska, M., von der Mosel, H.: Regularizing and self-avoidance effects of integral Menger curvature. Ann. Sci. Norm. Super. Pisa Cl. Sci. (5) 9(1), 145-187 (2010)

[20] Sullivan, J.M.: Approximating Ropelength by Energy Functions. AMS Contemporary Mathematics, Providence (2002)

[21] Sutton, A.P., Balluffi, R.W.: Interfaces in Crystalline Materials. Monographs on the Physics and Chemistry of Materials. Oxford University Press, USA, 2 (1997)

[22] Tolsa, X.: Analytic capacity, rectifiability, and the Cauchy integral. In: International Congress of Mathematicians, vol. II, pp. 1505-1527. European Mathematical Society, Zürich (2006) 